\title{
A Deep Learning Network for Right Ventricle Segmentation in Short-Axis MRI
}

\author{
Gongning Luo ${ }^{1}$, Ran $\mathrm{An}^{1}$, Kuanquan Wang ${ }^{1 *}$, Suyu Dong ${ }^{1}$, Henggui Zhang ${ }^{1,2}$ \\ ${ }^{1}$ Harbin Institute of Technology, Harbin, China \\ ${ }^{2}$ University of Manchester, Manchester, UK
}

\begin{abstract}
The segmentation of the right ventricle (RV) myocardium on MRI is a prerequisite step for the evaluation of $R V$ structure and function, which is of great importance in the diagnose of most cardiac diseases, such as pulmonary hypertension, congenital heart disease, coronary heart disease, and dysplasia. However, RV segmentation is considered challenging, mainly because of the complex crescent shape of the RV across slices and phases. Hence this study aims to propose a new approach to segment $R V$ endocardium and epicardium based on deep learning. The proposed method contains two subtasks: (1) localizing the region of interest (ROI), the biventricular region which contains more meaningful features and can facilitate the $R V$ segmentation, and (2) segmenting the $R V$ myocardium based on the localization. The two subtasks are integrated into a joint task learning framework, in which each task is solved via two multilayer convolutional neural networks. The experiments results show that the proposed method has big potential to be further researched and applied in clinical diagnosis.
\end{abstract}

\section{Introduction}

The cardiac diseases get the more attention of researchers in recent years, due to that the cardiac disease is the leading killer of human being[1, 2]. In the actual clinical diagnosis, the function indexes of heart are important for physicians. The function indexes, such as the end-diastole volumes (EDV), the end-systole volumes (ESV), and the eject fraction (EF), can be estimated through the segmentation method. However, most of researches focus on the left ventricle (LV) segmentation because the LV is the biggest chamber of heart [3-5]. Compared to the $\mathrm{LV}$, the segmentation problems of $\mathrm{RV}$ get more and more attentions in recent years, along with the increasing cognition about the function of RV [6-8]. For instance, some cardiac diseases such as pulmonary hypertension, cardiomyopathy and dysplasia, are all related to the RV.

In the actually clinical diagnosis of cardiac diseases, the cardiac magnetic resonance (CMR) is an important imaging modality and widely used because of its special advantages such as non-invasive detection, low radiation doses and high imaging quality [9]. Generally speaking, CMR is considered as gold standard in cardiac disease diagnosis especially in LV and RV function estimation. Hence, in this paper, the main modality of this research is CMR.

Compared to LV, the RV segmentation problem has more difficulties, for instance, the complex crescent shape, the presence of trabeculation and the relatively thinner wall of ventricle. Hence, after the RV segmentation workshop of MICCAI 2012 [6], comparatively few works focus on the RV segmentation. The methods of LV segmentation can be classified into two kinds: the methods based on traditional image processing and the methods based on deep learning.

In the term of traditional image processing, [10] proposed a method based on a combination of a novel window-constrained accumulator threshold technique, which incorporated a priori segmentation window constraints to guide and refine RV segmentation. [11] proposed a novel RV segmentation method based on moving mesh correspondences technology, which can be used as automatic propagation of the right ventricle (RV) endocardial and epicardial boundaries in 4D (3D+time) CMR sequences to achieve the RV segmentation in 4D CMR datasets. Besides, the proposed method in [11] can also be extended to be applied in RV congenital heart disease datasets.

In terms of deep learning, inspired by the powerful representation ability of convolutional neural networks (CNN) [12-14], some researchers proposed RV analysis methods based on CNN. [15] proposed a directed RV volumes prediction method based on CMR using the CNN and random forests. [16] proposed a fully convolutional neural network for cardiac segmentation in short-axis CMR, which can handle the RV and LV segmentation problems simultaneously. [17] proposed a method based on CNN and stacked autoencoder, which is trained to locate the region of interest (ROI) and delineate the boundary of RV chamber.

Additionally, the related review papers [18, 19] summarize more methods in new views. In this paper, inspired by the paper [20], we proposed a new method to 


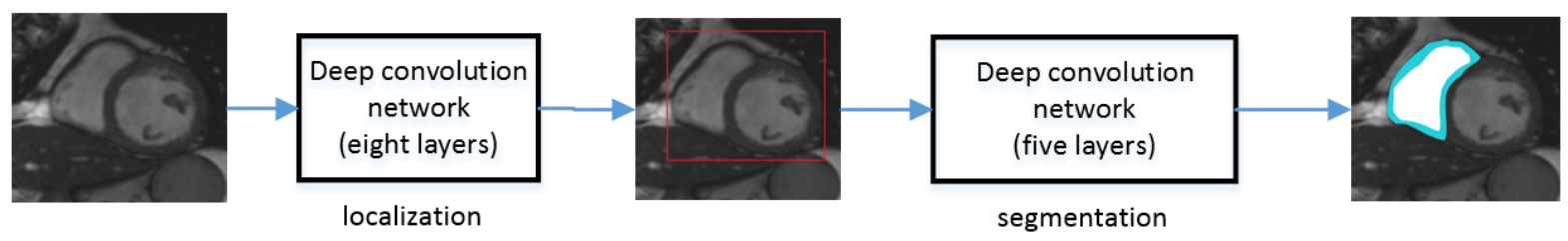

Figure 1. The framework of the proposed method.

address the RV segmentation problems in CMR using deep CNN networks. The rest of this paper is organized as follows. In the section 2, we introduced the basic frameworks about the proposed methods, including the location of ROI and RV segmentation based on deep CNN. In the section 3, we showed the results of RV segmentation experiments. Finally, we concluded this paper in the section 4 .

\section{Methods}

In the field of computer vision, the image segmentation problems are often divided into two subtasks: the location of ROI and the objects segmentation to get the final pixel-wise object masks. Inspired by the traditional natural images segmentation methods, we proposed a new framework to address RV segmentation problem in the field of medical image processing. The Figure 1 shows the basic framework of the proposed method, the details can be introduced in the following sections.

\subsection{Location of ROI}

Firstly, to achieve accurate and robust ROI location model in the first task, we selected 960 CMR images with manual labelled squares, whose sizes are $150 * 150$. We generated the squares through labelling the center points and cropping the ROI around the center points. Because the task of location aims at the slices across the whole short-axis, hence these manual labelled CMR images are the base slices in short-axis. Additionally, the manual labelled squares contain the RV and LV, because some experiments have proved that such regions can be located more accurately than the regions only containing the RV.

As shown in the Figure 1, we constructed the eight layers fully convolutional networks to achieve the location of ROI. We formulated the location problems into following cost function:

$$
F(R O I)=\frac{1}{2 N} \sum_{i=1}^{N}\left(\left|X^{t}-X\right|^{2}+\left|Y^{t}-Y\right|^{2}\right)+\frac{\lambda}{2}\|W\|^{2}
$$

where the $X^{t}$ denotes the relative position of the center points in horizontal direction. The $Y^{t}$ denotes the relative position of the center points in vertical direction. $W$ denotes the weights of the filters. Note that the number of filters is 6 in the every convolutional layer and the size of the convolutional kernel is $32 * 32 . N$ and $i$ denote the size of training sets and subject number respectively. The $\lambda$ denotes the weight coefficient of regular term.

To achieve relatively faster location processing, we proposed a transfer squares method based on the relationships across the slices along the short-axis (apex, mid, and base slices). Additionally, we find that most of subjects exist little shift among slices. Hence, as shown in Figure 2, the red square in the base slice can be easily transferred into the mid and bottom slices vertically. Finally, the ROI in every short-axis slice can be got.

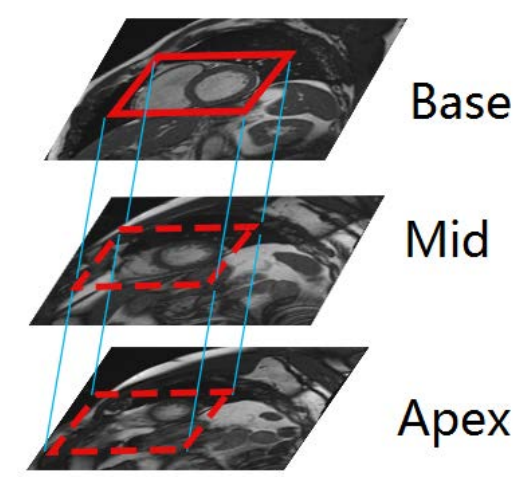

Figure 2. The transfer method in the location of ROI

\subsection{RV segmentation}

The location of ROI can improve the computation efficiency because the following segmentation processing can focus on the ROI with $150 * 150$ size.

As shown in the Figure 1, the deep convolutional networks for endocardium and epicardium segmentation of RV consist of five fully convolutional layers. In every layer, the number of filters is five, and the size of every kernel is $31 * 31$. The parameters of the two networks are optimized via back propagation. The loss function of the optimization is:

$$
F(\operatorname{seg})=\frac{1}{2 N} \sum_{i=1}^{N}\left(\left|V^{t}-V\right|^{2}\right)+\frac{\lambda}{2}\|W\|^{2}
$$

where $N$ refers to the amount of training examples, $V^{t}$ denotes the ground truth mask vectors of $\mathrm{RV}$ and $V$ denotes prediction mask vectors. The $i$ denotes the subject 
number. Additionally, the image with masks are expressed into vectors through unrolling the original images. $W$ denotes the weights of the filters and the $\lambda$ denotes the weight coefficient of regular term.

Compared to the traditional hand-crafted features, the main advantage of deep learning is that it can learn some representational features automatically. Furthermore, to avoid the ambiguity of objects mask, trainings of segmentation of endocardium and epicardium are independent.

The model can be optimized using stochastic gradient descent (SGD). In this paper, the training is conducted for 300 iterations. We selected the best segmentation model at the minimum loss value of validation datasets (the 332 slices including 200 training slices and 132 validation slices) during the training process as the finial segmentation model. This distribution method of training and validation data enhanced the generalization ability of the final segmentation model.

\section{Results and discussion}

\subsection{Datasets and metrics}

The deep learning network was trained and validated on cardiac MRI datasets from MICCAI 2012 right ventricle segmentation challenge including 48 patients (16 training patients and 32 test patients), which are the biggest RV datasets to my best knowledge. In the step of training ROI location model, we manually labelled the base slices in short-axis of the 48 patients for training location model. In the step of segmentation, we adopted the ground truth (486 slices from 16 training patients across different positions along the short-axis) from RV segmentation workshop of MICCAI 2012 to train and test the segmentation model. Note that the 486 slices from 16 train patients are divided into the training sets including 332 slices and testing sets including 154 slices.

At last, the proposed method was evaluated by the recognized criterions, including Dice metric (DM), Hausdorff distance (HD), correlation coefficient (R) and mean errors (ME) for end-diastole volumes (EDV), endsystole volumes (ESV) and ejection fraction (EF). Note that the EDV and ESV are got by summing the volumes in each slice on end-diastole and end-systole phases. The volumes in each slice are computed as the product of the corresponding cavity area (the endocardium segmentation results) and the slice thickness.

\subsection{Environment of experiments}

We conducted the experiments in the workstation, whose configurations are $3.4 \mathrm{GHz}$ Core i7 CPU, 64GB RAM, Nvidia TiTan X (12GB memories). The codes are based on python language and Keras deep learning framework. The learning ratio of SGD is 0.0001, the batch size is 12 in per iteration of training, the number of iteration is 300 for training the RV segmentation model and ROI location model.

\subsection{Prediction accuracy}

The results by comparison with ground truth are as following: Endocardium: $\mathrm{DM}=0.86 \pm 0.09, \mathrm{HD}=6.9 \pm$ 2.6mm; Epicardium: $\mathrm{DM}=0.84 \pm 0.13, \mathrm{HD}=8.9 \pm 5.7 \mathrm{~mm}$; EDV: $\mathrm{R}=0.89, \mathrm{ME}=7.1 \pm 4.5 \mathrm{ml} ; \mathrm{ESV}: \mathrm{R}=0.84, \mathrm{ME}=9.6$ $\pm 6.7 \mathrm{ml}$; $\mathrm{EF}: \mathrm{R}=0.86, \mathrm{ME}=7.5 \pm 5.3 \%$. Besides, as shown in Figure 3, the proposed model has fast convergence rate especially at the start of training. The power fitting of the trend of loss decrease also is shown in

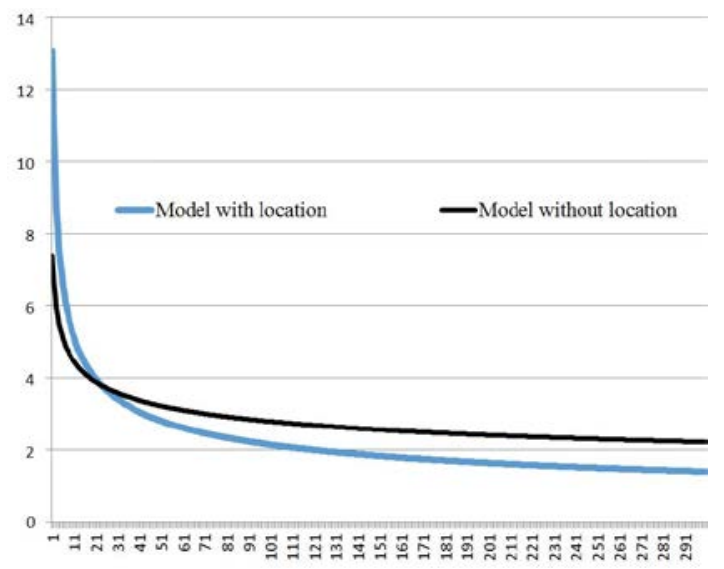

Figure 3. The trend line of the loss decrease.

Figure 3, which shows the good reduce trend of the proposed prediction model.

\subsection{Efficiency}

We report the efficiency of the proposed method in terms of ROI location, the number of CNN layers and model training.

- $\quad$ Some experiments without the ROI location step were also attempted. As we can see in the Figure 3, the trend line of loss decrease prove that the step of location can achieve relatively faster convergence and higher accuracy. Generally, the cost of time in location is one hour for training and one second for computation. Compared with the improvement in the term of accuracy, this cost is worth.

- In the term of CNN networks, the number of CNN layers is the leading factor for computation cost. After a lot of attempts with different depth of the CNN, we found the eight CNN layers in the location step and five CNN layers in the segmentation step are good balance between computation efficiency and location accuracy. Besides, the designed deep CNN can achieve 5s 
and $2 \mathrm{~s}$ in every iteration of training in location and segmentation steps respectively.

\section{Conclusion}

In this paper, we proposed a new RV segmentation method based on multi-tasks framework using deep learning technology. Specially, the proposed ROI location method improve the segmentation accuracy and computation efficiency in some extent. The final segmentation results prove that the proposed method has big potential to be further researched and applied in clinical diagnosis.

\section{Acknowledgements}

This work is supported by the National Natural Science Foundation of China (NSFC) under Grant No. 61571165 and No. 61572152. The Titan X used for this research was donated by the NVIDIA Corporation. Thanks for MICCAI 2012 RV segmentation Challenge, who provided the CMR data for this research work.

\section{References}

[1] Selvanayagam JB. Non-Invasive Cardiac Imaging: Past, Present and Future. Heart, lung \& circulation. 2016;25(8):755-6.

[2] Luo GN, Sun GX, Wang KQ, Dong SY, Zhang HG. A novel left ventricular volumes prediction method based on deep learning network in cardiac MRI. Computing in Cardiology: IEEE; 2016.

[3] Wang LJ, Pei MC, Codella NCF, Kochar M, Weinsaft JW, Li JQ, et al. Left Ventricle: Fully Automated Segmentation Based on Spatiotemporal Continuity and Myocardium Information in Cine Cardiac Magnetic Resonance Imaging (LV-FAST). Biomed Res Int. 2015.

[4] Dong SY, Luo GN, Sun GX, Wang KQ, Zhang HG. A left ventricular segmentation method on 3D echocardiography using deep learning and snake. Computing in Cardiology: IEEE; 2016.

[5] Dong SY, Luo GN, Sun GX, Wang KQ, Zhang HG. A combined multi-scale deep learning and random forests approach for direct left ventricular volumes estimation in 3D echocardiography. Computing in Cardiology: IEEE; 2016.

[6] Petitjean C, Zuluaga MA, Bai WJ, Dacher JN, Grosgeorge $\mathrm{D}$, Caudron J, et al. Right ventricle segmentation from cardiac MRI: A collation study. Medical Image Analysis. 2015;19(1):187-202.

[7] Bogachkov A, Ayache JB, Allen BD, Murphy I, Carr ML, Spottiswoode B, et al. Right ventricular assessment at cardiac MRI: initial clinical experience utilizing an ISSENSE reconstruction. The international journal of cardiovascular imaging. 2016;32(7):1081-91.

[8] Caudron J, Fares J, Lefebvre V, Vivier PH, Petitjean C, Dacher JN. Cardiac MRI Assessment of Right Ventricular Function in Acquired Heart Disease:Factors of Variability. Acad Radiol. 2012;19(8):991-1002.
[9] Suinesiaputra A, Medrano-Gracia P, Cowan BR, Young AA. Big Heart Data: Advancing Health Informatics Through Data Sharing in Cardiovascular Imaging. Ieee J Biomed Health. 2015;19(4):1283-90.

[10] Ringenberg J, Deo M, Devabhaktuni V, Berenfeld O, Boyers P, Gold J. Fast, accurate, and fully automatic segmentation of the right ventricle in short-axis cardiac MRI. Comput Med Imag Grap. 2014;38(3):190-201.

[11] Punithakumar K, Noga M, Ben Ayed I, Boulanger P. Right ventricular segmentation in cardiac MRI with moving mesh correspondences. Comput Med Imag Grap. 2015;43:15-25.

[12] Zhang L, Yang F, Zhang YD, Zhu YJ. Road crack detection using deep convolutional neural network. IEEE International Conference on Image Processing (ICIP 2016)2016.

[13] Shin HC, Roth HR, Gao M, Lu L, Xu Z, Nogues I, et al. Deep Convolutional Neural Networks for Computer-Aided Detection: CNN Architectures, Dataset Characteristics and Transfer Learning. IEEE Trans Med Imaging. 2016;35(5):1285-98.

[14] Krizhevsky A, Sutskever I, Hinton G E. Imagenet classification with deep convolutional neural networks. Advances in neural information processing systems 2012. p. 1097-105.

[15] Zhen X, Wang Z, Islam A, Bhaduri M, Chan I, Li S. Multiscale deep networks and regression forests for direct biventricular volume estimation. Med Image Anal. 2016;30:120-9.

[16] Phi VT. A Fully Convolutional Neural Network for Cardiac Segmentation in Short-Axis MRI. arXiv. 2016.

[17] MR Avendi, Arash Kheradvar, Jafarkhani H. Fully automatic segmentation of heart chambers in cardiac MRI using deep learning. $\mathrm{J}$ Cardiovasc Magn $\mathrm{R}$. 2016;18(Suppl1):P351.

[18] Petitjean C, Dacher JN. A review of segmentation methods in short axis cardiac MR images. Medical Image Analysis. 2011;15(2):169-84.

[19] Peng P, Lekadir K, Gooya A, Shao L, Petersen SE, Frangi AF. A review of heart chamber segmentation for structural and functional analysis using cardiac magnetic resonance imaging. Magma. 2016;29(2):155-95.

[20] Wang X, Zhang L, Lin L, Liang Z, Zuo W, editors. Deep joint task learning for generic object extraction. Advances in Neural Information Processing Systems; 2014.

Address for correspondence.

Kuanquan Wang

Mailbox 332, Harbin Institute of Technology

Harbin 150001, China

wangkq@hit.edu.cn 